\title{
ANTROPOLOGía PEDAGÓGICA, FIN PARA LA EDUCACIÓN
}

\section{Javier Bermejo Fernández-Nieto}

\section{RESUMEN}

La Antropología de la Educación apareció, en nuestro país, en los planes formativos para las carreras universitarias del Magisterio a finales del siglo pasado y a pesar de su carácter, peso e interés, no ha permanecido vigente tras la última reconstrucción de planes realizada hace unos años. Este hecho no ha sucedido en otros países europeos como es el caso concreto de Alemania, cuna originaria del estudio antropológico-filosófico, donde, debido a su amplia tradición, goza de un gran prestigio tanto en los programas académicos como en la extensa bibliografía científica existente sobre la misma. Este artículo pretende ensalzar la importancia de la atención a la Antropología Pedagógica desde los planes de estudios en la formación de maestros partiendo de la tesis fundamental de que para la tarea educativa es necesario disponer de un foco antropológico sustancial que facilite la comprensión del ser humano y que fundamente la posibilidad del fenómeno educativo.

Palabras clave: antropología pedagógica, antropología filosófica, desarrollo de la persona, formación de maestros.

\section{TITLE: PEDAGOGICAL ANTHROPOLOGY END FOR EDUCATION}

\section{ABSTRACT}

The Educational Anthropology appeared last century in the formative plans for university Magisterium courses. Despite its character, weight and interest, it hasn't remained in force after the plans reconstruction was made a few years ago. This fact hasn't appeared in other European countries such as Germany, home of anthropological-philosophical study, where because of its rich tradition, enjoys a great prestige in academic programs such as its extended scientific literature. This article aims to extol the Pedagogical Anthropology attention importance from the curriculum in teacher training based on the fundamental thesis that educational work is necessary to have a substantial anthropological focus which makes easier the understanding of human beings and substantiate the possibility of educational phenomenon.

Keywords: Pedagogical Anthropology, Philosophical Anthropology, Individual Development, Teacher Training.

Javier Bermejo Fernández-Nieto < javiberme@hotmail.com > . Departamento de Teoría e Historia de la Educación (Universidad Complutense de Madrid) / Dirección particular: Avenida Baunatal 19, I B, 28703, San Sebastián de los Reyes (Madrid). Original recibido: I5-06-I5. Original aceptado: 2I-I2-15. 


\section{Introducción}

El término Antropología dispone de multitud de usos dentro del lenguaje común y científico. Como queda patente dentro del elenco de ciencias, el estudio del ser humano es coincidente y recursivo para multitud de disciplinas. La biología, sin ir más lejos es el mejor y más perceptible ejemplo pues estudia la vida y, con ella y en ella, al ser humano. La psicología, por citar otro ejemplo, también estudia al ser humano en su conjunto centrando la atención en su psiquis, en la implicación de ésta en la conducta. Por su parte, la Antropología Pedagógica, objeto de este ensayo, parte de su raíz originaria, siendo esta la Antropología Filosófica. En términos consecuentes y partiendo de lo anteriormente citado, puede extrapolarse que la Antropología Filosófica como ciencia estudia el fenómeno humano en toda su integridad o dicho de otro modo, la Antropología Filosófica centra su atención de manera radical en las características que posibilitan dicho fenómeno. Su esencia es por tanto el estudio del ser humano en cuanto tal.

Para poder focalizar la ingente tarea filosófica que la materia dispone, proponemos tratar su conceptualización en términos hermenéuticos. Para comprender las implicaciones del todo -Antropología- en las partes y viceversa, se hace necesario atender de manera primigenia al hecho de que el ser humano es gracias a lo que hace y es capaz de hacer. Por ello, lo que hace, en todas sus formas y posibles, así como lo que es capaz de hacer, configuran la esencia propia de su ser. En efecto, en primer término, para saber lo que es el ser humano, es necesario saber lo que el hombre hace y lo que puede llegar a hacer. Este aspecto, de forma inexorable, es una exhortación a la educabilidad, a la capacidad humana de educación, pues entre otros aspectos, el ser humano es capaz de educarse, de educación.

Siguiendo nuestra aproximación, la Antropología Pedagógica pretende ser un acercamiento reflexivo a la realidad humana a modo de instrumento teórico para abordar con sustancialidad y sentido la tarea educativa. A nuestro parecer, no es plausible realizar una íntegra y adecuada práctica educativa sin una aproximación real de lo que es el ser humano y de lo que puede llegar a ser, y este aspecto no puede ser atendido únicamente por medio de ciencias de cohorte biológico, pues, como es más que constatable, el ser humano no es solo en la medida de sus posibles biológicos sino en la medida en que dichos posibles se configuran o engranan con otros. Así, esta materia surge dentro de la reflexión filosófica de la Antropología, concretamente en la tradición filosófica germana, de forma paralela y sumatoria 
gracias a las aportaciones de diversos autores, principalmente estadounidenses. Es por ello por lo que la Antropología Filosófica forma el eje sobre el que la Antropología Pedagógica encuentra su lugar en el estudio del ser humano.

En síntesis, dado su origen y escenario de capacidades, entendemos que la Antropología Pedagógica -o de la Educación- debe disponer de un interés particular para aquellas personas que van a dedicarse al ejercicio de tareas educativas. Su encuadre, lejos de reducirse a lo planteado por diversos autores, no se centra en exclusividad en la mera transmisión cultural', sino en la atención a todas las dimensiones del ser humano. No se pretende aquí enumerar ni las temáticas ni los más que sabidos beneficios de esta materia sino analizar su importancia en lo que a la educación se refiere, itiene sentido su desaparición del corpus de asignaturas que configuran las ciencias de la educación? ¿Es la Antropología de la Educación un fin para la propia educación? Vamos a analizarlo.

\section{El hombre como ser educable}

Comienza Marco Aurelio sus Meditaciones haciendo acopio de sus propias virtudes, agradeciendo a cada uno de sus antepasados la enseñanza de las mismas. De su abuelo paterno el buen carácter y la serenidad, de su padre el carácter discreto y viril, de su madre el respeto a los dioses y la generosidad, de su preceptor la imparcialidad y de su bisabuelo la preocupación por la educación. Tal vez, el emperador no destacó de forma arbitraria estos atributos, ni ciertamente los ordenó al azar, lo que sí queda patente es que ante todo se mostraba agradecido a todos ellos por la transmisión recibida y el poso que en él había quedado sobre cada una de las virtudes reseñadas. Sólo una reflexión interior, comedida, y fruto de la doctrina socrática -conócete a ti mismo- pudo dar lugar a ello.

El ser humano es el único animal que no nace entero, ni termina nunca de enterarse. Así podríamos decir que el inacabamiento humano está presente de forma constatada pues el ser humano es capaz de ser más y mejor, en suma, es capaz de crecer sin límites ni techo: "más somos los humanos cuanto más y mejor conocemos, y cuanto más y mejor queremos” (Barrio, 2013, p. I62). El ser humano es capaz de crecer gracias a las expectativas de potencialidad que la educación tiene de él y únicamente gracias a una educación fundamentada este ser humano puede llegar a desarrollarse de forma sustancial. Es bien sabido que la educación tiene por motivo 
el crecimiento de la persona más allá de los posibles elementales pertenecientes a la raza humana como especie, como son el raciocinio o el lenguaje. Tal vez no debiéramos obviar este último aspecto, el lenguaje. La perspectiva del ser humano como ser capaz de lenguaje, como ser gramático puede colaborar a estructurar una primera base antropológica lo suficientemente sólida como para plantear a continuación nuevos escenarios de aprendizaje y perfeccionamiento así como formas o modelos pedagógicos mejorados (López Herrerías, 200I). El lenguaje pudiera ser el primer aspecto, o el más elemental del ser humano. Si algo diferencia a éste del resto de animales es el lenguaje. Otros animales son capaces de comunicarse, incluso de vivir de forma conjunta (manada), pero no son capaces de lenguaje ni de cultura como tal. Tal como describe Arendt (1992), mediante las palabras y los actos nos insertamos en el mundo humano y comenzamos a estimularnos al interactuar con los demás. El lenguaje es, así, nuestro primer medio de socialización, de apertura y presentación con el mundo al que llegamos.

Dentro del proceso educativo, a su vez, entran en valor diversas consideraciones como son el momento histórico en el que se desarrolle el mismo, el entorno, su cultura, etc. Pero no menos importante es el educador, encargado de desarrollar y mantener el proceso educativo abierto de forma consecuente, cabal y razonado.

Con todo, sostener que el ser humano es un ser educable supone, consecuentemente, comprender qué es el ser humano y qué puede llegar a ser (García Amilburu, 1996). La relación entre Educación y Antropología es más que evidente. En todo modelo educativo subyace un modelo antropológico, así, la especificidad del ser humano como ser cultural manifiesta de forma directa la vinculación entre la antropología y la educación.

Dentro de este marco, el concepto de educabilidad es el más recurrente en los manuales de Antropología Pedagógica como eje argumental de las posibilidades que el ser humano tiene con la educación. Si bien es cierto, no es hasta el primer tercio del siglo $X X$, gracias a $M$. Scheler, cuando se vira sustancialmente entorno a la diferencia entre el animal y el hombre. En su obra El puesto del hombre en el cosmos, el filósofo alemán arguye su Antropología Filosófica incidiendo en el hecho de que la diferencia fundamental entre ambos es el espíritu, cuya esencia definía como la facultad de desligarse de la presión biológica. Así las cosas, un ser con espíritu no está inexorablemente encadenado a sus instintos, o a su medio, sino que es capaz de elevar el medio a la objetividad, en palabras del propio filósofo: "El hombre puede 
reprimir y someter los propios impulsos; puede rehusarles el pábulo de las imágenes perceptivas y de las representaciones. Comparado con el animal, que dice sí siempre a la realidad, incluso cuando la tema y la rehúye, el hombre es el ser que sabe decir no, el asceta de la vida, el eterno protestante contra toda mera realidad" ( I 94 I, p. 85-86). Siguiendo esta premisa, no es la razón lo que nos diferencia de los animales, sino el espíritu. En adición, y ahondando más en esta cuestión, resulta más que interesante la siguiente afirmación de $M$. Buber: "No se trata de considerar la razón como lo específicamente humano $y$, por el contrario, lo que en el hombre no es racional, como lo no específico, lo que comparte con seres no humanos... El hombre no es un centauro sino íntegramente hombre” (201 I , p. 80)². Esta afirmación, además de contundente, deja entrever que somos lo que somos mas siendo íntegramente hombres, disponemos de una característica propiamente humana, la anteriormente mencionada educabilidad.

Gracias a la misma, y por mediación de su potencialidad de crecimiento, el ser humano es educable, es mejor-able. En sentido estricto, siguiendo esta premisa, podríamos decir que es mejorable como ser en tanto en cuanto es capaz de educación: su espíritu es mejorable.

La educabilidad como concepto surge o se nutre de la capacidad humana para el aprendizaje. En este sentido, y apoyándonos en Gehlen (1986), podríamos indicar que entran en juego dos aspectos a nuestro juicio fundamentales, a saber: aplicación y disponibilidad. El proceso vital humano presenta una oportunidad innegable hacia el crecimiento. Éste, mediado por el aprendizaje dispone de un alcance operativo y funcional que posibilita a la persona para objetivar su medio y potenciar su desarrollo. En consonancia, se trata de otorgar valor al desarrollo humano -crecimientosin obviar la premisa fundamental que Gehlen expone: el hombre ante todo es un ser con condición de carencia. En este sentido, su crecimiento y desarrollo, consecuentemente, permite minimizar las mismas.

\section{La Antropología Pedagógica en el eje educativo}

En lo que a materia de Antropología Pedagógica se refiere, es la corriente alemana contemporánea -Roth, Dienelt, Langeveld o Hamman entre otros- la más involucrada en la importante tarea de integrar la amplia tradición científica en consonancia con los estudios abordados en materia antropológico-pedagógica. 
Tal vez por su cercanía en el discurso, Bruno Hamman ha sido uno de los autores más estudiados en nuestro país. Para el alemán existen tres concepciones antropológicas fundamentales en la modernidad, a saber: la occidental-cristiana, la natural-biologista y la marxista (Hamann, 1992). Siguiendo este orden, la primera concepción antropológica centra su foco en el ser humano como homo sapiens, una suma de materia y espíritu. Por su parte, la concepción biologista contempla al hombre como homo faber, capaz de dominación, y por último, la marxista presenta una imagen materialista del hombre, quien sólo adquirirá valor en la medida en que sea capaz de liberarse de las relaciones alienantes del trabajo productivo. Una revisión bibliográfica sobre la materia pone de manifiesto que cada época histórica ha presentado fundamentalmente una concepción concreta del hombre, no en vano la reflexión filosófica entorno al hombre siempre ha permanecido como constante.

Cabría resolver la cuestión ¿Es la Antropología de la Educación un medio o un fin para la actividad educativa? Como refleja el profesor David Reyero (2000) pudiera conceptualizarse ésta como fin de la filosofía de la educación y, al mismo tiempo, podría concebirse como medio para conseguir una sólida base teórica que fundamente su ejercicio. Más allá de la constatable tradición en la documentación germana, la Antropología Pedagógica extendida en todo el mundo, demuestra un interés patente. Si acudimos a buscadores generalistas en red, como por ejemplo Google, apreciamos más de 120.000 resultados para los términos "antropología pedagógica", asimismo se obtienen 472.00 resultados para las palabras "antropología de la educación". Estos datos muestran que las alusiones a esta terminología están lejos de ser menores ${ }^{3}$.

\section{La Antropología Pedagógica como fundamentación del ejercicio del magisterio}

En este sentido, se hace necesario revisar someramente la realidad de los planes formativos actuales en lo que al magisterio se refiere. En nuestro país, la tendencia hacia la diversificación y especialización ha ido creciendo de forma paralela al resto del continente, si bien es cierto tal vez nunca se haya llegado a alcanzar ese nivel de especificidad que en otras regiones europeas se ha conseguido. Como todo proceso, auge o potencialidad, la excesiva especialización ha ido mermando en detrimento de la generalidad $y$, tras la inclusión de los nuevos planes universitarios, 
se ha abogado por reducir la amplísima oferta de asignaturas -tal vez excesiva- sin atender a las necesidades o requerimientos de cada carrera universitaria. Una vez más, la experiencia nos puede sugerir que ante los extremos lo mejor es la mesura. Como bien señala el Catedrático de Teoría de la Educación, Gonzalo Jover (2013), postulado ya el Espacio Europeo de Educación Superior, se hace necesario abogar por una Sociedad de la Sabiduría que ayude y posibilite conseguir profesionales competentes, afianzando los principios teóricos y éticos ya alcanzados, sin olvidar el hecho de que las competencias no pueden reducirse, ni definirse, únicamente desde los requerimientos laborales, pues dejarían de responder a los requerimientos de una formación integral.

La educación es educarse, así tituló H-G. Gadamer (2000) su conferencia en el Dietrich-Bonhoeffer-Gymnasium de Eppelheim en 1999, en la que ponía de manifiesto que el objetivo fundamental de la educación es que el hombre acceda a él mismo. Para ello, entran en juego multitud de aspectos y figuras, como son, la cultura y los educadores, los educandos y el entorno, mas a nuestro juicio no es posible educarse -en los términos que Gadamer nos interpela- sin disponer de un concepto propio, humano, tácito. En efecto, la educación es un proceso atemporal mediante la cual el ser humano consigue desarrollarse como persona. Dentro del crecimiento humano, $y$ en lo que a sus dimensiones se refiere, es posible diferenciar dos aspectos sustanciales: el crecimiento intelectual y el moral, así como las dimensiones: social, afectiva y religiosa o del sentido (Barrio, 2004). Para que se produzca una verdadera educación todas las dimensiones anteriormente indicadas han de ser atendidas, pues de no ser así, no podría afirmarse que el acto educativo atiende a la radicalidad del ser humano. En cualquier caso, el mero hecho de intentar definir con cierta profundidad las características de un modelo filosófico-antropológico, requiere de un extenso espacio de páginas, es por ello, por lo que atenderemos aquí, partiendo de la premisa gadameriana de aproximarnos a nuestra propuesta sin olvidar que el objeto de este ensayo es la necesidad de vincular estrechamente el modelo antropológico al ejercicio educativo.

Como bien nos trasladaba el Catedrático de Filosofía de la Educación, Quintana Cabanas (2012) en su último libro Ni lobo ni cordero. El hombre es un ser mejorable, la educación tiene como objetivo fundamental el diálogo con los temas más elementales del ser humano. De poco o nada sirve, -según Quintana- contemplar al ser humano como lobo o como cordero si no se abordan respuestas o se confrontan 
los pormenores de cada posible concepción humana. Sírvanos esta obra como sustento de nuestro argumento: ante la tesis y la antítesis, siempre cabe esperar una síntesis. El ser humano es capaz de sustentar su postura vital en cualquiera de los extremos posibles, por ello, la educación debe procurar, acompañar y guiar a los educandos hacia la síntesis anteriormente mencionada. En este sentido la Antropología de la Educación tiene mucho que decir. Más allá de la aportación Filosófica, el hecho de poner el foco en la Antropología, repercute de manera directa en los posibles del hombre y por extensión en lo que a la educación de los mismos se refiere. La premisa de H-G. Gadamer parece descargar toda responsabilidad en el propio sujeto, haciéndole algo más que simple partícipe de su desarrollo humano, no obstante, el imperativo al que alega el alemán centra más su atención en la temporalidad -prolongación en el tiempo- que en el simple hecho de condecorar el acto educativo y centralizarlo en la persona como única responsable. La educación es, en efecto, mucho más que atemporal. La educación es para la persona, y para la persona en el tiempo, por ello, primigeniamente es necesario conceptualizar a la misma como objeto base del acto educativo, como realidad provista de otra realidad -realidad personal, realidad educativa-. El ser humano, indica Spaemann (2002), es sustancia viva y por ende realidad propia. Esta realidad repleta de estados es capaz de educación y así mismo la educación es para el ser humano. Podríamos por tanto decir que cada una de ellas es una realidad consecuente para con la otra. $Y$ toda esta consecuencia no puede sino concebirse dentro de un marco históricopedagógico, en los términos en los que C. Wulf (2006) describe, a saber: el hombre sólo puede ser comprendido en un contexto histórico -temporal y social- en este caso, educativamente.

Por su parte, no podemos obviar la razón de ser de las facultades humanas, pues a fin de cuentas son las que posibilitan la propia educación. La existencia de estas facultades en nosotros viene exigida por epor la existencia de congruencia entre las operaciones que realizamos y los principios próximos a ellas. Así, si las operaciones están en el plano accidental deben situarse también en el plano potencial ya que la educación centra su fundamentación en las potencias operativas del ser humano (García López, 1986). Por todo ello, el ejercicio educativo puede asemejarse a un arte, puesto que se interesa en la mirada del otro y hacia el otro (Freitag, 20I2). El ejercicio del magisterio, lejos de su concepción romántica, es un alegato al ser humano, pues nace de él para él. Qué duda cabe que al no tratarse de una actividad 
puramente técnica, la educación puede erigirse como un acto vocacional. Atendiendo a este aspecto, las dimensiones vocacionales del ejercicio del magisterio han sido descritas por diversos autores, existiendo un cierto volumen bibliográfico en torno a ello, no obstante, nos gustaría destacar dos de las más significativas: ser arquitecto y la atención a los detalles (Hansen, 200I). Con todo, el docente que dispone de ambas dimensiones, pudiéramos decir, que goza de un amplio y ventajoso terreno ganado para desarrollar con competencia su ejercicio. No es fácil proyectar un estereotipo plasmado en un papel a la misma realidad, si bien es cierto, el arquitecto contemplará todos los pros y los contras de su edificación, de su proceso, de los materiales necesarios, de su temporalidad y fases. Una vez confeccionado el plano, comenzará la ejecución de su proyecto. Del mismo modo, deberá cuidar cada detalle, por minúsculo que fuere, para que su proyecto vea la luz con el éxito que merece. Lo mismo sucede con la educación y su ejercicio.

\section{Futuro requerido}

Como se ha puesto de manifiesto en las líneas anteriores, la educación en general y los educadores en particular están llamados a facilitar, favorecer y proporcionar crecimiento humano en todas sus dimensiones, no sólo técnicas sino reflexivas, personales y humanísticas (Derrick, 20 I I). En este sentido, se aboga aquí por luchar contra la inercia de las modas, en este caso, educativas.

En efecto, la propuesta aquí recogida se sintetiza en la consideración necesaria de conceptualizar pedagógicamente al ser humano de forma integral más allá de los planes formativos del Magisterio que en nuestro país han omitido, curricularmente hablando, los programas de Antropología de la Educación, o Antropología Pedagógica ${ }^{4}$.

Por otro lado la tarea educativa requiere de compromiso mutuo. Dicho de otro modo, el educador y el educando han de establecer una relación educativa de forma bidireccional en aras de posibilitar verdaderamente el acto educativo, mas ambos deben tomar parte en y durante el transcurso de éste. Así, la neutralidad desaparece pues no es posible entablar una relación educativa si ninguna de las partes rompiese esa neutralidad (Ollero, 200I). Ante la importantísima tarea que los docentes tienen tanto con el presente como con el futuro de nuestras sociedades, creemos necesario superar, en cierta medida, la crisis actual en favor del ser humano. En una sociedad cada vez más desfragmentada, y a la vez rica en diversidad, se hace 
necesario otorgar cierto valor antropológico a nuestras actividades (Noguera, 1995), y no podemos obviar que la educación es algo más que una simple actividad al uso. Si no queremos mimetizar lo que de manera plástica denominaba C.S. Lewis como mono con pantalones ${ }^{5}$ tal vez debiéramos interpelar a la realidad educativa actual. Esa interpelación debiera acompañarse de una conceptualización antropológica capaz de colaborar con el enriquecimiento de la propia educación para no limitarse únicamente a la mera transmisión de competencias, pues como se ha expresado con anterioridad, el ser humano no sólo es capaz de técnica aunque ésta albergue o contemple gran parte de los procesos mediados de aprendizaje. El corpus teórico en el ámbito educativo se integra por la aportación de varias ciencias y entre ellas la Antropología facilita dos asuntos fundamentales: "no perder de vista que el protagonismo en la educación lo tiene cada persona, para lo que hay que contemplar su unidad y complejidad al mismo tiempo, y que esta perspectiva conduce a la interdisciplinariedad y a la necesidad de vislumbrar, desde un punto de vista integrador, la complementariedad de los conocimientos científicos de áreas diversas que nos aportan datos relevantes para asumir la educación" (Bernal, 2006, pp.165-166). Abordar la cuestión educativa es imposible sin referirse a un anthropos o realidad humana, que por otra parte, $y$ tal como se manifiesta líneas más arriba, es una realidad inacabada. Bajo esta realidad, las consecuencias derivadas de la ausencia de formación antropológica en los planes de Magisterio son claras: no resolver el problema de la persona, o al menos no abordarlo en forma teórica ${ }^{6}$. En efecto, transmitir fundamentos educativos a los futuros profesionales de la educación requiere, al menos, de conocer no sólo los fundamentos, sino la fundamentación teórica de la persona humana en cuanto tal. ¿Qué es el hombre?, ¿qué es la educación?, ¿qué dimensiones tiene el ser humano? Etc. En suma, se trata de transmitir consciente y consecuentemente partiendo de la persona y para la persona. En primera instancia, y apoyándonos en Millán-Puelles (1975), cabe destacar que en el orden antropológico la pregunta por la persona se estructuraría del siguiente modo: idónde radica la dignidad del hombre? En segundo lugar, y nuevamente trayendo a Spaemann (2005) a nuestro escrito, podríamos decir que hablar de conciencia es hablar de la dignidad del hombre. En suma conocer al hombre es conocer cómo se estructura su conciencia para construir su dignidad.

La educación tiene mucho trabajo por delante, y sus profesionales también. El valor que la Antropología Pedagógica dispone, creemos es más que evidente, 
por ello, el trabajo sobre dicha materia en cuanto a su investigación, reflexiones y conceptualizaciones continuará, aun a sabiendas de su desaparición dentro de los programas formativos.

\section{Conclusiones finales}

Algo tan común, tan corriente y a la vez tan complejo como es el hecho de vivir en sociedad, dispone de diversas maneras de ser asimilado, y requiere de un aprendizaje específico para ello. Tal vez este sea el argumento básico sobre el que se sustenta la necesidad de atención de manera considerada hacia la educación. Es ella y mediante ella -entre otras cosas- desde la cual el sencillo o complejo hecho de vivir en sociedad pudiera ejercerse de modo cívico y cabal. En este sentido, qué duda cabe que la cultura y sus procesos juegan un papel importante en el desarrollo personal, pero, no son los únicos elementos que forman parte del juego.

Para que la educación surja dentro de unos límites coherentes, en primera instancia se hace necesario atender a la misma desde una concepción antropológica. Actualmente la educación, en lo que a su enseñanza se refiere, goza de un amplio espectro de asignaturas centradas en la ejecución técnica en detrimento de las enseñanzas teóricas o filosóficas. Así, los planes actuales de Pedagogía en nuestro país atienden sobre todo al ser humano, en lo que a su carácter biológico se refiere, y al estudio de su potencialidad psíquica, pero la formación científica y pedagógica de los/as profesionales de la educación, a nuestro juicio, no puede restringirse a una capacitación puramente técnica, sino más bien reflexiva. Es conveniente -y más considerando la desaparición de esta materia en el corpus de asignaturas universitarias del magisterio- recuperar para éstas la reflexión antropológico-filosófica, sin obviar ni olvidar, por supuesto, la dimensión técnica y tecnológica de la educación, pero siendo conscientes de que ante todo el ejercicio educativo es una tarea humana y humanística.

Se hace necesario, por tanto, potenciar la reflexión sustancial en el alumnado de Magisterio y Pedagogía. Es muy lícito y resulta inevitable, incluso fundamental, que las personas nos hagamos preguntas y tratemos de resolverlas, pero no es menos necesario el que éstas tengan cierta presencia e importancia interior y no se limiten a resolver los embarazos diarios de la cotidianidad. Lo mismo sucede con la educación. Ésta debe resolver sus cuestiones más elementales y a su vez las más complejas. El ser 
humano es capaz de educación y análogamente la educación es capaz de aprehensión por el ser humano. Así, la Antropología de la Educación, aun habiendo desaparecido del panorama universitario actual, continuará aportando su inmenso valor en la reflexión filosófica de nuestros días y por extensión, permanecerá intrínsecamente dentro de las lecciones magistrales de nuestro profesorado universitario. Es por ello por lo que vindicamos la necesidad de atender a la dimensión antropológica en la formación de los profesionales de la educación, pues consideramos que no es posible realizar una tarea educativamente sustancial sin disponer de un concepto del ser humano más allá de lo que desde la biología o la psicología se refiere. Aquí es donde radica la afirmación de que la Antropología es un fin para la educación.

El análisis filosófico desde la perspectiva antropológico-pedagógica, tiene como objeto la propuesta de modelos antropológicos plurales que sean capaces de componer y estructurar un escenario favorable para el desarrollo educativo de las personas y que a su vez sea capaz de atender a la diversidad existente en la actualidad, sirviendo sobre todo de base para la tarea educativa. Esta pluralidad, entendemos, debe partir y converger fundamentalmente en el hecho de conocer a la persona y sus dimensiones fundamentales desde una perspectiva ética y responsable. Así, dentro del abanico amplio de concepciones resulta indispensable contextualizar en primer lugar al ser humano, sin escepticismos, y consecuentemente conceptualizarlo pedagógicamente -sin relativismos-. En adición, la tarea pedagógica hoy, está más que nunca llamada a la reflexión filosófica en pro del ser humano, de su crecimiento y sobre todo de su educación.

\section{Referencias bibliográficas}

Arendt, H. (1993). La condición humana. Barcelona: Paidós.

Barrio Maestre, J. Ma. (2004). Elementos de Antropología Pedagógica. Madrid: Rialp.

Barrio Maestre, J. Mª (2013). El Dios de los filósofos. Curso básico de filosofía. Madrid: Rialp.

Bernal Martínez de Soria, A. (2006). Antropología de la educación para la formación de profesores. Educación y Educadores, 9 (2), I49-167. 
Buber, M. (20I I). ¿Qué es el hombre? México: Fondo de Cultura Económica.

Derrick, C. (20II) Huid del escepticismo. Una educación liberal como si la verdad contara para algo. Madrid: Encuentro.

Freitag, V. (20I2). El arte al encuentro de la antropología: reflexiones y diálogos posibles. Praxis \& Saber, 3 (6), | |2|-|40.

Gadamer, H-G. (2000). La educación es educarse. Barcelona: Paidós.

Gehlen, A. (1986). Antropología filosófica. Del encuentro y descubrimiento del hombre por sí mismo. Barcelona: Paidós.

García Amilburu, Ma. (1996). Aprendiendo a ser humanos. Una Antropología de la Educación. Pamplona: EUNSA.

García López, J. (1986). El sistema de las virtudes humanas. México: ERM.

Hamann, B. (1992). Antropología Pedagógica. Introducción a sus teorías, modelos y estructuras. Barcelona: Vicens Vives.

Hansen, D. T. (200I). Llamados a enseñar. Barcelona: Idea Books.

Jover Olmeda, G. (2013). Recreación del Espacio Europeo de Educación Superior en el horizonte de la Sociedad de la Sabiduría: hacia un nuevo escenario docente. Teoría de la Educación: Educación y Cultura en la Sociedad de la Información, I4 (3), 5-24.

Lewis, C. S. (1990). La abolición del hombre. Madrid: Ediciones Encuentro.

López Herrerías, J. A. (200I). El saber hacer pedagógico desde la categoría antropológica de la gramaticalidad. Revista Española de Pedagogía, 220, 483-502.

Marco Aurelio (2003). Meditaciones, Barcelona: Biblioteca de los grandes pensadores.

Millán-Puelles, A. (1975). El problema ontológico del hombre como criatura, Scripta Theologica, 7 (I), 309-333.

Noguera, J. (Ed.) (1995). Cuestiones de Antropología de la Educación. Barcelona: CEAC.

Ollero, A. (200I). Democracia y convicciones en una sociedad plural. Pamplona: Cuadernos Instituto Martín de Azpilcueta. 
Reyero, D. (2000). ¿Es la antropología el final de la filosofía de la educación?, Enrahonar: Quaderns de filosofía, 31, 95-105.

Scheler, M. (194I). El puesto del hombre en el cosmos. Losada: Buenos Aires.

Spaemann, R. (2002). Realidad como antropomorfismo. Anuario Filosófico, 35, 713730.

Spaemann, R. (2005). Ética: cuestiones fundamentales. Pamplona: EUNSA.

Quintana Cabanas, J. Ma . (20I2). Ni lobo ni cordero. El hombre es un ser mejorable. Barcelona: AEAU.

Wulf, C. (2006). Antropología histórico-cultural de la educación. Estudios Filosóficos, 55 (160), 449-466.

\section{NOTAS}

I. Hacemos alusión a propuestas que centran, casi en exclusividad, su atención en el ejercicio y posibles de la transmisión de cultura como eje fundamental de la tarea educativa. Propuesta que inicialmente pudiera entenderse como reduccionista.

2. La concepción antropológica de Buber centra su atención en la afirmación de que en el hombre existe una parte perteneciente a su naturaleza como ser vivo -animaly por otro lado que no todo lo humano tiene condición animal como tal. Como el mismo afirma: "Ni siquiera el hambre de un hombre es el hambre de un animal" (p.80).

3. Centramos nuestra atención en el motor de búsqueda estadounidense. En este sentido, utilizando su herramienta específica para material académico (Google Académico), los resultados también reflejan importancia en volumen: 19.800 para la búsqueda "antropología pedagógica" y 38.600 para "antropología de la educación". Consultado el I0. II. 2015.

4. Del total de universidades españolas que disponen de programas de grado vinculado a la educación (un total de 67), la Antropología de la Educación como 
materia continúa únicamente en los planes de 16 de ellas, vinculándose en muchas ocasiones a otros estudios y ciencias como la filosofía, la sociología o las bases de investigación educativa.

5. Nos referimos a la expresión del escritor inglés utilizada en su famosa obra La abolición del hombre (1990) en la que pone de manifiesto su rechazo al subjetivismo y mediante la cual aboga por la necesidad de que el ser humano, ante todo, no se rechace a sí mismo.

6. Centramos aquí la atención en la concepción sobre el problema de la persona en los términos expresados por Millán-Puelles, entendiendo el concepto bajo la tridimensionalidad del mismo, a saber: antropológica, metafísica y teológica. Todas ellas buscan dar respuesta fundamentada a la relación entre la persona y el resto de conceptos. 